\title{
Nevus de Becker
}

\author{
José Luis Fortes Álvarez ${ }^{\mathrm{a}}$
}

\section{a Médico de Familia. \\ Consultorio de Saceruela.}

EAP Abenójar. Ciudad Real.

\section{Correspondencia:} José Luis Fortes Álvarez. C/ Grecia $n^{\circ} 27$ 13005 - Ciudad Real. Tfno: 926232991.

Recibido el 6 de septiembre de 2007.

Aceptado para su publicación el 2 de octubre de 2007.

\section{RESUMEN}

Son muchas las lesiones cutáneas de color marrón, de diferentes tonos, que nos pueden alarmar a los médicos de familia, pensando en diagnósticos de mal pronóstico. El Nevus de Becker es una de estas lesiones, que a modo de máculas se presenta en personas jóvenes, preferentemente varones, que suelen consultar por el problema estético que suponen, pues la mayoría de las veces son asintomáticas. En cuanto al diagnóstico diferencial, lo tenemos que hacer con otras enfermedades de la piel, del tipo de nevus melanocitico congénito, neurofibromatosis (mancha de café con leche) y amiloidosis cutánea, entre otras.

Palabras clave. Nevus, melanosis.

\section{ABSTRACT}

\section{Becker's Nevus.}

There are many skin lesions that are of different shades of brown. This could alarm family doctors who may diagnose a disease with poor a prognosis. Becker's Nevus is one of these lesions and it usually occurs in young people, especially males. It appears has a brown macule on the skin and is mainly symptom-free. Patients usually go to the doctor for aesthetic reasons. A differential diagnosis must be made with other skin diseases such as congenital melanocytic nevus, neurofibromatosis, and cutaneous amyloidosis amongst others.

Key Words. Nevus, melanosis.

\section{INTRODUCCIÓN}

El nevus de Becker es considerado como un auténtico nevus epidermico, y así lo confirma la anatomía patológica, sin embargo otros autores lo engloban dentro de los hamartomas y como tal hay que considerarlo. Presenta una evolución benigna, por lo que se recomienda observación y revisiones periódicas ante posibles cambios en el desarrollo del paciente.

\section{OBSERVACIONES CLÍNICAS}

Presentamos el caso de un paciente de 21 años que consulta por presentar una lesión de color marrón claro en región pectoral desde hace un año. Ha sido valorado previamente por su médico de familia, que no le ha dado importancia, recomendándole observación periódica y volver a consultar, en el caso de aparecer cambios clínicos, para ser remitido al Dermatólogo. La preocupación principal del joven, por lo que solicita la consulta del Dermatólogo, es porque coincidiendo con la época estival le ha aparecido pelos de distribución atípica sobre la mancha, con el problema estético que esto supone.

\section{DISCUSIÓN}

El nevus de Becker se encuentra dentro de los nevus epidérmicos desde el punto de vista histológico, pudiendo aparecer en edades tempranas, como la infancia o la pubertad. Predomina en varones, por lo que se cree que los andrógenos juegan un papel importante en su desarrollo, además de asociarse frecuentemente con hi- 
pertricosis, según va evolucionando a lo largo de los años. En un estudio reciente, Nirdé et al pusieron en evidencia esta asociación entre el Nevus de Becker y la hipersensibilidad a los andrógenos. Otros autores afirman que se trata de un hamartoma, y como tal hay que considerarlo.

Con frecuencia una exposición solar es la desencadenante de su aparición en la piel. La forma inicial de presentación es una mácula pigmentada de color marrón de bordes mal definidos, levemente verrugosa, siendo frecuente su localización en zona escapular, pecho, dorso de extremidad superior y hombro. No suele asociarse a prurito. La ubicación es unilateral y con el paso del tiempo la lesión macular se llena de pelos pudiendo desplazar el área de pigmentación. Ro et al describieron un caso de Nevus lineal que se distribuyó desde el hombro derecho, siguiendo las líneas de Blaschko, poblándose de pelos a los 4 años.

Se caracteriza histológicamente por un aumento del tamaño de los melanocitos localizados en la capa basal, sin aumentar el número de los mismos, además del incremento del número de dendritas y de una marcada hiperpigmentación de toda la epidermis. Ocasionalmente podemos ver asociaciones con otras lesiones dermatológicas, así Puri et al describieron un caso raro de nevus de Becker con liquen plano, coincidiendo los dos diagnósticos en la misma zona de piel.

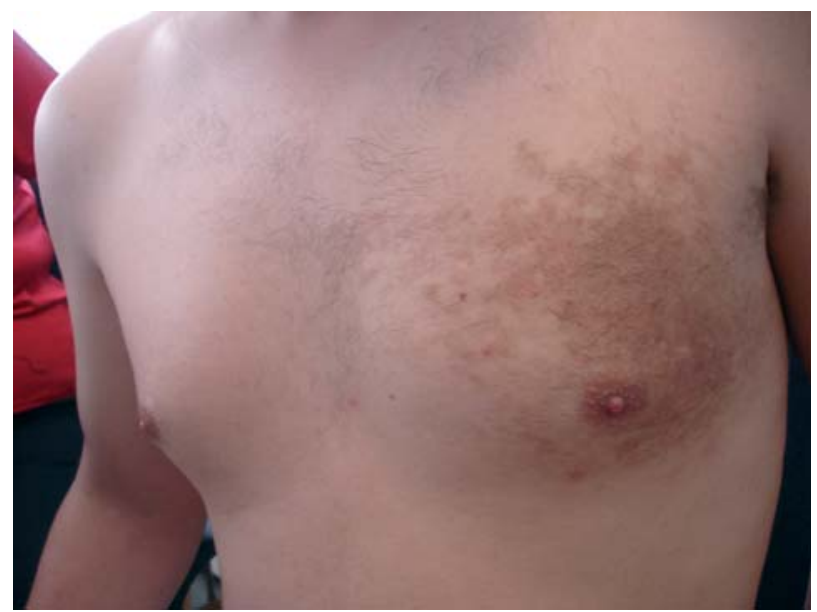

Figura 1. Nevus de Becker.

\section{BIBLIOGRAFÍA}

1. Ingordo V, lannazzone SS, Cusano F, Naldi L. Dermoscopic features of congenital melanocytic nevus in an adult male population: an analysis with a 10-fold magnification. Dermatology 2006; 212(4):354-60.

2. Puri S, Nanda S, Grover C, Batra VV, Garg VK, Reddy BS. Congenital Becker nevus with lichen planus. Pediatr Dermatol 2005; 22(4):328-30.
3. Ro YS, Ko JY. Linear congenital Becker nevus. Cutis 2005; 75(2):122-4.

4. Nirdé P, Dereure O, Belon C, Lumbroso S, Guilhou JJ, Sultan C. The association of Becker nevus with hypersensitivity androgens. Ach Dermatol 1999; 135(2):212-4. 